\title{
Entorhinal Cortical Deep Brain Stimulation Rescues Memory Deficits in Both Young and Old Mice Genetically Engineered to Model Alzheimer's Disease
}

\author{
Frances Xia ${ }^{1,2,9}$, Adelaide Yiu ${ }^{2,9}$, Scellig SD Stone ${ }^{3,4}$, Soojin Oh $^{2}$, Andres M Lozano ${ }^{5,6}$, Sheena A Josselyn ${ }^{*, 1,2,7,8}$ \\ and Paul W Frankland*,1,2,7,8 \\ 'Department of Physiology, University of Toronto, Toronto, ON, Canada; ${ }^{2}$ Program in Neurosciences \& Mental Health, Hospital for Sick Children, \\ Toronto, ON, Canada; ${ }^{3}$ Harvard Medical School, Boston, MA, USA; ${ }^{4}$ Department of Neurosurgery, Boston Children's Hospital, Boston, MA, USA; \\ ${ }^{5}$ Division of Neurosurgery, Department of Surgery, University of Toronto, Toronto, ON, Canada; ${ }^{6}$ Toronto Western Research Institute, Krembil \\ Discovery Tower, University Health Network, Toronto, ON, Canada; ${ }^{7}$ Department of Psychology, University of Toronto, Toronto, ON, Canada; \\ ${ }^{8}$ Institute of Medical Sciences, University of Toronto, Toronto, ON, Canada
}

\begin{abstract}
Alzheimer's disease (AD) is a neurodegenerative disorder characterized by progressive cognitive decline. Deep brain stimulation (DBS) has been used to treat a variety of brain disorders and shows promise in alleviating cognitive symptoms in some AD patients (Laxton et al, 2010). We previously showed that DBS of the entorhinal cortex (EC) enhances spatial memory formation in normal (wild-type) mice (Stone et al, $201 \mathrm{I}$ ). Here we tested the effects of EC-DBS on the progressive cognitive deficits in a genetically-based mouse model of AD. TgCRND8 (Tg) transgenic mice express human amyloid precursor protein harboring the Swedish and Indiana familial AD mutations. These mice exhibit age-related increases in A $\beta$ production, plaque deposition, as well as contextual fear and spatial memory impairments. Here, we found EC stimulation in young mice (6 weeks old) rescued the early contextual fear and spatial memory deficits and decreased subsequent plaque load in Tg mice. Moreover, stimulation in older mice (6 months old) was also sufficient to rescue the memory deficits in Tg mice. The memory enhancement induced by DBS emerged gradually (over the course of weeks) and was both persistent and specific to hippocampal-based memories. These results provide further support for the development of novel therapeutics aimed to resolve the cognitive decline and memory impairment in AD using DBS of hippocampal afferents.

Neuropsychopharmacology (2017) 42, 2493-2503; doi:I0.1038/npp.2017.100; published online 21 June 2017
\end{abstract}

\section{INTRODUCTION}

It is predicted that by $2050>131.5$ million people worldwide will have dementia (Prince et al, 2016). Alzheimer's disease (AD), the most common form of dementia, was first described by Alois Alzheimer in 1906. Despite intense research in the over 100 intervening years, there is no cure for $\mathrm{AD}$ and the basic underlying pathophysiological mechanisms are not well understood. The defining feature of $\mathrm{AD}$ is memory loss, associated with dysfunction of the medial temporal lobe, including the hippocampus (Perry and Hodges, 1996, Albert, 2011). The amyloid beta hypothesis of $A D$ posits that $A D$ results from abnormal accumulation of the amyloid beta protein $(A \beta$, derived from the proteolytic processing of amyloid precursor protein, APP) (Tanzi and

*Correspondence: Dr SA Josselyn or Dr PW Frankland, Program in Neurosciences and Mental Health, Hospital for Sick Children, 555 University Avenue, Toronto, ON M5G IX8, Canada, Tel: +(4I6) 8I37654, E-mail: sheena.josselyn@sickkids.ca or paul.frankland@sickkids.ca ${ }^{9}$ These authors contributed equally to this work.

Received 24 February 2017; revised 16 May 2017; accepted 18 May 2017; accepted article preview online 25 May 2017
Bertram, 2001, Selkoe, 2002). Although challenged (Morris et al, 2014), this hypothesis remains influential. In particular, autosomal dominant mutations in APP cause familial AD (fAD) (Citron et al, 1992, Price and Sisodia, 1998, Hardy and Selkoe, 2002).

Currently, there are two approved pharmacological treatments for the cognitive symptoms of $\mathrm{AD}$, cholinesterase inhibitors and NMDA receptor antagonists. These drugs have limited efficacy and recent clinical trials for new therapies have been disappointing. Deep brain stimulation (DBS) is currently being used to treat several brain disorders, including Parkinson's disease, dystonia, and treatmentresistant depression (Mayberg et al, 2005, Ponce and Lozano, 2010). Recently, DBS has also been investigated as a possible treatment for patients with $\mathrm{AD}$, motivated by a case study in which DBS of the hypothalamus and fornix (the major output fiber bundle of the hippocampus) enhanced memory recollection (Hamani et al, 2008). During implantation surgery of a patient being treated for morbid obesity, acute DBS stimulation of the hypothalamus/fornix induced an episodic memory-like phenomenon (the patient recalled a familiar scene). Furthermore, turning on stimulation of the 
implanted electrode 2 months post surgery similarly evoked a memory-like phenomenon. This patient also showed enhanced memory recall following 3 weeks of chronic stimulation. A Phase 1 study with six participants with early-stage $\mathrm{AD}$ was conducted (Laxton et al, 2010). Six months of chronic stimulation decreased cognitive decline (as measured on standardized tests) in four of six patients and all patients showed sustained increases in glucose metabolism (measured by PET) across multiple brain regions including the hippocampus, parahippocampal gyrus and entorhinal cortex. A follow-up Phase II study conducted in 42 patients with early $\mathrm{AD}$, which used a 'stimulation on' $v s$ 'stimulation off cross-over design, showed no overall cognitive benefit produced by 12 months of continuous stimulation (although there was a tendency for slowing the cognitive decline in a subset of patients) (Lozano et al, 2016). However, increases in glucose metabolism in brain regions including the hippocampus and several cortical regions were noted following 6 (but not 12) months of stimulation. Together these initial findings hint at the promise of DBS for treating the memory-related symptoms of $\mathrm{AD}$, but also indicate that further basic research is necessary to inform future clinical trials.

We previously showed that acute DBS of the entorhinal cortex (EC), a region afferent to the hippocampus, produced pro-cognitive effects in adult wild-type (WT) mice (Stone et al, 2011). Six weeks following acute DBS, mice showed improved spatial memory, as assessed in the water maze. Here we test whether a similar intervention reverses the memory deficits and plaque load in mice genetically engineered to model AD.

\section{MATERIALS AND METHODS}

\section{Mice}

TgCRND8 (Tg) mice express a human $\mathrm{APP}_{695}$ transgene with both the Swedish (K670N-M671L) and Indiana (V717F) fAD mutations under the regulation of the Syrian hamster prion promoter (PrP) (Chishti et al, 2001) and were obtained from Dr David Westaway (University of Alberta, Canada). Tg mice were maintained in a 129S6/SvEvTac genetic background and crossed with WT C57BL/6NTac mice to produce F1 hybrid experimental mice. Tg and WT littermate control mice were either 6 weeks (young mice) or 6 months (old mice) old at the start of all experiments. Mice were group housed (3-5 mice per cage) on a 12-h light/dark cycle. Behavioral experiments were conducted during the light phase. Food and water were available ad libitum. Approximately equal numbers of male and female mice were examined. All procedures were conducted in accordance with policies of the Hospital for Sick Children Animal Care and Use Committee and conformed to both Canadian Council on Animal Care (CCAC) and National Institutes of Health (NIH) Guidelines on Care and Use of Laboratory Animals.

We stimulated TgCRND8 mice at two ages (6 weeks and 6 months). We chose 6 weeks as the earliest age because the TgCRND8 is an accelerated model of AD (Chishti et al, 2001). In these mice, memory deficits are evident at 6 weeks of age, and plaques emerge at $\sim 10-12$ weeks of age (Yiu et al, 2011). Stimulating mice at 6 weeks of age allowed us to assess the effect of DBS when memory deficits first emerge (and before significant plaque deposition). At 6 months of age, these $\mathrm{Tg}$ mice also show memory deficits but also significant plaque load.

\section{Stereotactic Surgery and EC stimulation}

Bilateral EC stimulation was conducted as previously described (Stone et al, 2011). Six-week- or 6-month-old mice were pre-treated (atropine sulfate $0.1 \mathrm{mg} / \mathrm{kg}$, i.p.), anesthetized (chloral hydrate, $400 \mathrm{mg} / \mathrm{kg}$, i.p.) and placed in a stereotaxic frame. Concentric bipolar electrodes (FHC, CBA SC75) were bilaterally targeted to the EC (coordinates relative to Bregma in the anteroposterior, mediolateral, and dorsoventral planes as follows (in $\mathrm{mm})$ : $(-4.0, \pm 3.25,-5.1)$, Paxinos and Franklin, 2001). Electrical stimulation was designed to approximate high frequency DBS used in clinical practice (Volkmann et al, 2006), and delivered (via a clinical screener, model 3628; Medtronic; frequency $=130 \mathrm{~Hz}$, pulse width $=90 \mu \mathrm{s}$, square wave) for $1 \mathrm{~h}$ in the $\mathrm{Tg}$ stimulated group (Tg-S). Control, non-stimulated groups (WT-NS, TgNS) were implanted with electrodes bilaterally in the EC, but did not receive stimulation. Behavior experiments were conducted 1, 3, or 6 weeks post stimulation.

\section{Contextual and Auditory Tone Fear Conditioning and Testing}

Contextual fear training. Contextual fear training consisted of placing a mouse in the conditioning chamber and $2.5 \mathrm{~min}$ later, delivering a foot shock $(2 \mathrm{~s}, 0.5 \mathrm{~mA})$. The mouse remained in the training context for an additional $30 \mathrm{~s}$.

Contextual fear testing. Testing for contextual fear memory occurred $24 \mathrm{~h}$ and 1 week after conditioning (except where explicitly stated). Mice were returned to the training context for $5 \mathrm{~min}$. The amount of time spent freezing (defined as an immobilized, crouched position, with an absence of any movement except respiration (Blanchard and Blanchard, 1969) during context re-exposure was assessed. Freezing behavior was monitored by overhead cameras, which digitized video images at $4 \mathrm{~Hz}$. An automated frameby-frame analysis of movement was used to generate freezing scores (FreezeFrame software, Actimetrics, Wilmette, IL).

Tone fear training. Mice trained and tested 6 weeks after DBS were trained in auditory fear conditioning. Auditory tone fear conditioning consisted of placing mice in a conditioning chamber and, 2 min later, presenting a tone $(2800 \mathrm{~Hz}, 85 \mathrm{~dB}, 30 \mathrm{~s})$ that co-terminated with a foot shock $(2 \mathrm{~s}, 0.5 \mathrm{~mA})$. The mouse remained in the training context for an additional $30 \mathrm{~s}$.

Tone fear testing. Memory of the tone fear conditioning was assessed $48 \mathrm{~h}$ after training. Mice were placed in a novel chamber and, 2 min later, the tone was presented (for $1 \mathrm{~min}$ ). The amount of time spent freezing during tone presentation was assessed. 
Statistical analysis. The amount of time spent freezing when re-exposed to the training context (context fear) or tone presentation (tone fear) was compared across groups by an analysis of variance (ANOVA). Where appropriate, significant effects were further analyzed using NewmanKeuls post hoc tests.

\section{Water Maze Training and Spatial Memory Testing}

Training and testing in the water maze were conducted as previously described (Stone et al, 2011). The circular water maze tank $(120 \mathrm{~cm}$ diameter, $50 \mathrm{~cm}$ deep $)$ was located in a dimly lit room (see (Teixeira et al, 2006, Maei et al, 2009)). The pool was filled to a depth of $40 \mathrm{~cm}$ with water made opaque by nontoxic white paint. Water temperature was maintained at $28 \pm 1{ }^{\circ} \mathrm{C}$. A circular escape platform $(10 \mathrm{~cm}$ diameter) was submerged $0.5 \mathrm{~cm}$ below the water surface and located at a fixed position throughout training. The pool was surrounded by white curtains painted with distinct cues, $1 \mathrm{~m}$ from the pool perimeter.

Before training, mice were handled for $2 \mathrm{~min} /$ day for 1 week. Mice received three trials per day, for 6 days. Each trial lasted a maximum of $60 \mathrm{~s}$. To begin each trial, a mouse was placed in the pool, facing the wall in one of four start locations (varied pseudo-randomly). The trial was complete once the mouse found the platform or $60 \mathrm{~s}$ had elapsed. If the mouse failed to find the platform on any trial, the experimenter guided the mouse onto the platform. After each training trial, the mouse was allowed $15 \mathrm{~s}$ on top of the platform. After $60 \mathrm{~min}$ of the final training trial, spatial memory was assessed in a probe test during which the platform was removed from the pool and the mouse was allowed to search for $60 \mathrm{~s}$.

Training and probe trial behavioral data were acquired and analyzed using an automated tracking system (Actimetrics, Wilmette, IL). In training, latency to platform was measured and averaged over trials each day. In the probe test we quantified spatial memory by measuring the amount of time mice spent searching in the target zone $(20 \mathrm{~cm}$ radius, centered at the location of the platform during training; $11 \%$ of the pool surface) $v s$ the average time spent in the three other equivalent zones in other areas of the pool (Teixeira et al, 2006).

Statistical analysis. We analyzed time required to reach platform (escape latency) using a two-way ANOVA with between-group factor 'Group' and within-group factor 'Day' (6 days). For probe test, we first quantified spatial bias by comparing amount of time mice spent in target zone $v s$ average time spent in equivalent zones in 'other' three quadrants of the pool using an ANOVA (between-subjects variable vector, within-subjects variable zone (target, others)). Significant effects were further analyzed with Newman-Keuls post hoc tests.

\section{Plaque Immunostaining and Quantification}

Plaque immunostaining was conducted as previously described (Yiu et al, 2011). Coronal brain sections $(50 \mu \mathrm{m})$ from non-stimulated (Tg-NS) and stimulated (Tg-S) transgenic mice were treated with $1 \%$ hydrogen peroxide in $100 \%$ methanol (15 min), followed by $96 \%$ formic acid (10 min).

a
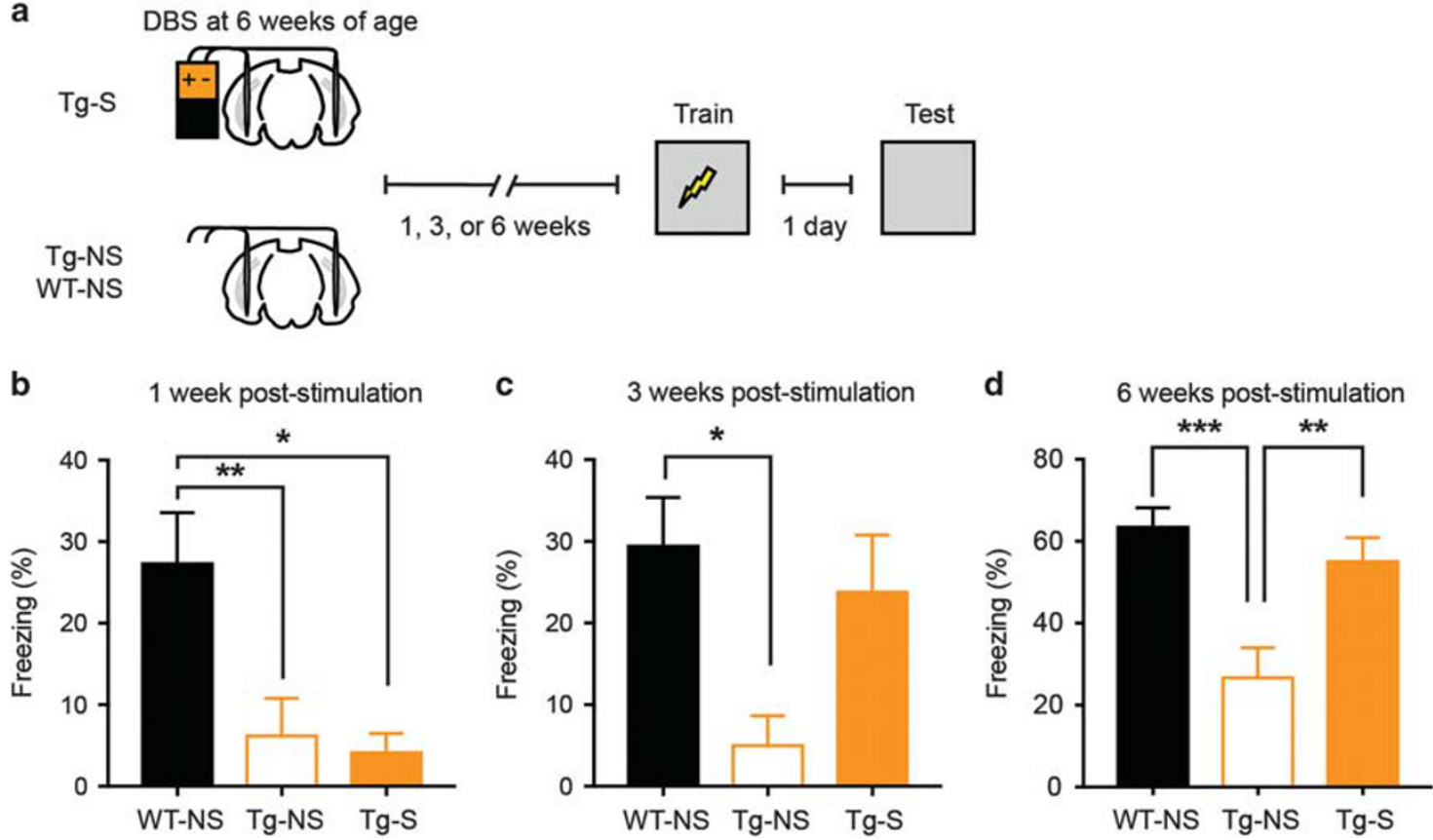

Figure I EC stimulation at 6 weeks of age rescues subsequent deficits in context fear memory in young Tg mice. (a) At 6 weeks of age, Tg mice were bilaterally stimulated in the EC (Tg-S) for I h, while Tg and WT controls were not stimulated (Tg-NS, WT-NS). Separate groups of mice were fear conditioned at I, 3, or 6 weeks post stimulation and tested $24 \mathrm{~h}$ later. (b) EC stimulated $\operatorname{Tg}(\operatorname{Tg}-\mathrm{S}, n=6)$ and control non-stimulated $\mathrm{Tg}(\mathrm{Tg}-\mathrm{NS}, n=8)$ mice trained I week post stimulation showed contextual fear memory deficit compared with non-stimulated WT-NS mice $(n=9)$. (c, d) Tg-S mice trained 3 weeks (WT-NS, $n=13$; Tg-NS, $n=8$; Tg-S, $n=11$ ) or 6 weeks (WT-NS, $n=25$; Tg-NS, $n=9$; Tg-S, $n=16$ ) post stimulation showed a rescue of context fear memory that was most prominent in mice trained 6 weeks after stimulation. Data are expressed as mean $\pm \mathrm{SEM}(* P<0.05, * * P<0.0 \mathrm{l}, * * * * P<0.00 \mathrm{I})$. 
a DBS at 6 weeks of age
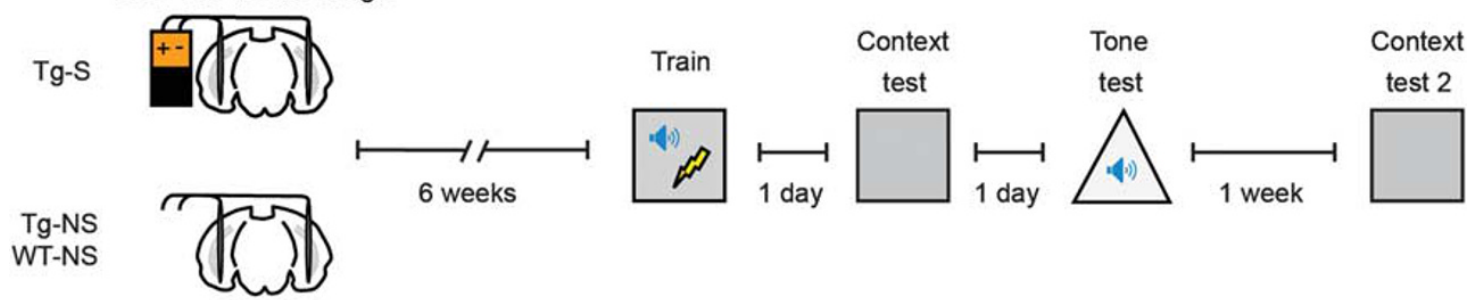

1 day

1 day
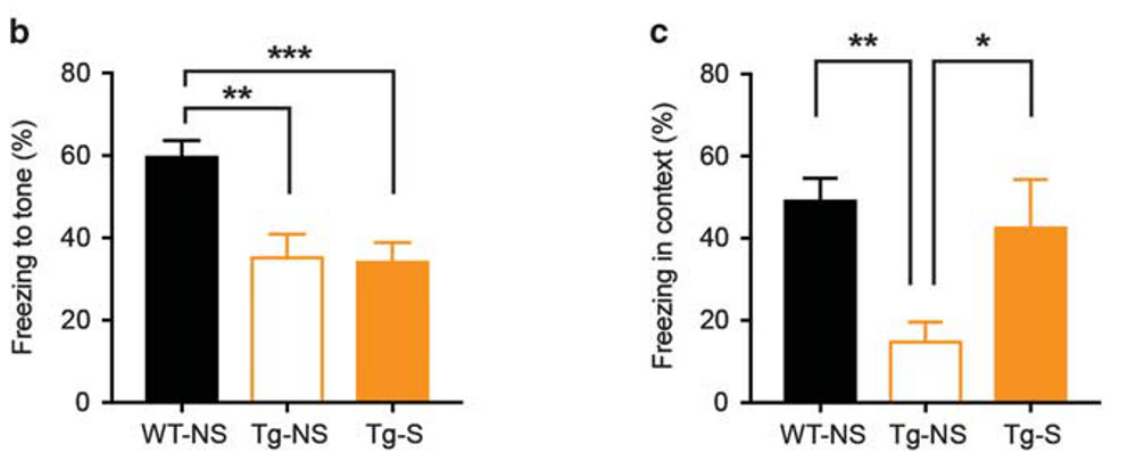

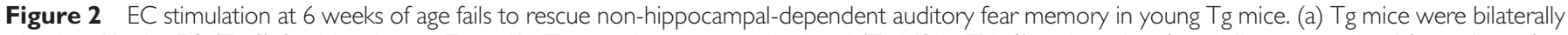

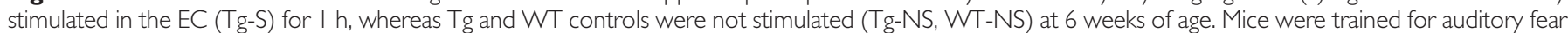

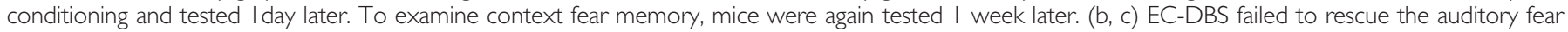

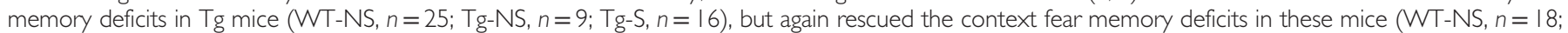

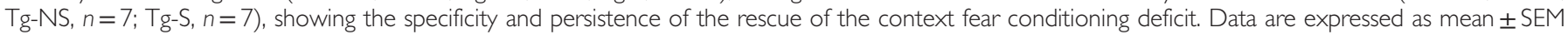
$(* P<0.05$, ** $P<0.01$, **** $P<0.001)$.

Plaques were stained with mouse anti-A $\beta-6 \mathrm{E} 10$ primary antibody (SIG-39320; 1:500, Covance, Emeryville, CA) followed by a biotinylated donkey anti-mouse secondary antibody (715-066-151; 1:500, Jackson ImmunoResearch, West Grove, PA) and visualized using avidin-biotin peroxidase coupled to DAB. For the 6 week-old $\mathrm{Tg}$ mice, the number of plaques was manually counted in the dorsal hippocampus and frontal cortex by two observers unaware of the treatment conditions. In 6-month-old $\mathrm{Tg}$ mice, plaques were dense and difficult to quantify using this method. Therefore, we instead quantified plaque area.

\section{RESULTS}

\section{EC Deep Brain Stimulation Reverses Contextual Fear Conditioning Deficits in Young TgCRND8 Mice}

We first tested whether EC-DBS affects the contextual memory impairments previously observed in young TgCRND8 mice (Steele et al, 2014). Six-week-old TgCRND8 mice received bilateral EC stimulation for $1 \mathrm{~h}(\mathrm{Tg}-\mathrm{S})$ or nostimulation (Tg-NS). One, 3 or 6 weeks later, stimulated and non-stimulated transgenic mice (Tg-S, Tg-NS), as well as age-matched, non-stimulated WT littermate control mice (WT-NS), were trained in contextually fear conditioning (Figure 1a). Contextual fear memory was assessed $24 \mathrm{~h}$ after training.

For mice trained 1 week following stimulation, both $\mathrm{Tg}$ groups (Tg-S and Tg-NS) froze considerably less than nonstimulated WT controls (Figure $1 \mathrm{~b} ; F_{2,20}=6.37, P<0.01$; WT-NS $(n=9)$, Tg-NS $(n=8), \operatorname{Tg}-\mathrm{S}(n=6))$. This finding confirms that this Tg AD model exhibits impaired context fear memory, even in young adulthood (Yiu et al, 2011).
Furthermore, these data indicate that contextual fear memory in $\mathrm{Tg}$ mice is unaltered 1 week following EC stimulation.

When separate groups of mice were trained 3 weeks following stimulation, a different pattern emerged. While unstimulated Tg mice (Tg-NS) froze less than WT control mice, stimulated $\mathrm{Tg}$ mice (Tg-S) froze at levels similar to WT mice (Figure 1c; $F_{2,29}=3.78, P<0.05$; WT-NS $(n=13)$, Tg-NS $(n=8)$, Tg-S $(n=11)$; post hocs Newman-Keuls). Mice trained 6 weeks following stimulation showed the same pattern of results. Again, stimulating $\mathrm{Tg}$ mice $(\mathrm{Tg}-\mathrm{S})$ increased freezing levels above their non-stimulated $\mathrm{Tg}$ littermates, similar to WT mice (Figure $1 \mathrm{~d} ; F_{2,47}=8.20$, $P<0.001$; WT-NS $(n=25)$, Tg-NS $(n=9)$, Tg-S $(n=16)$; post hocs Newman-Keuls). Together, these results indicate that acute deep brain stimulation of EC reversed the contextual fear memory deficits in young $\mathrm{Tg}$ mice. The benefits of DBS are not observed 1 week following stimulation, but emerge over a longer time course (ie, becoming evident 3 and 6 weeks post stimulation).

In the above group of mice trained 6 weeks following stimulation, a tone was paired with a foot shock during training. This provided an opportunity to assess tone fear conditioning, a form of learning that does not critically depend on the hippocampus (Kim and Fanselow, 1992), in these mice (Figure 2a). In contrast to contextual fear memory, EC stimulation failed to rescue tone fear memory in $\mathrm{Tg}$ mice (Figure 2b; $F_{2,47}=10.29, P<0.001$; WT-NS $(n=25)$ froze more than Tg-NS $(n=9)$ and Tg-S $(n=16)$ mice, which did not differ from each other, by Newman Keuls post hocs). One week later, we placed the same mice in the original training context to determine whether the 
a

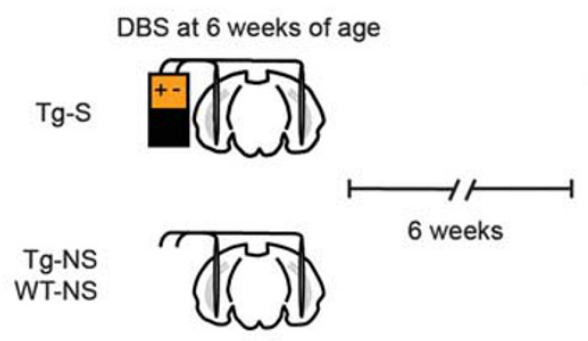

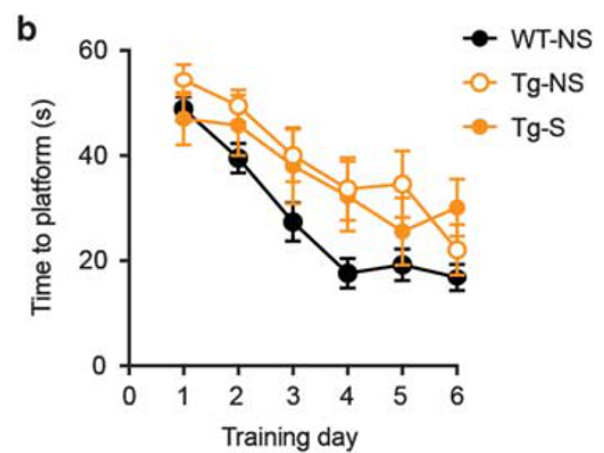
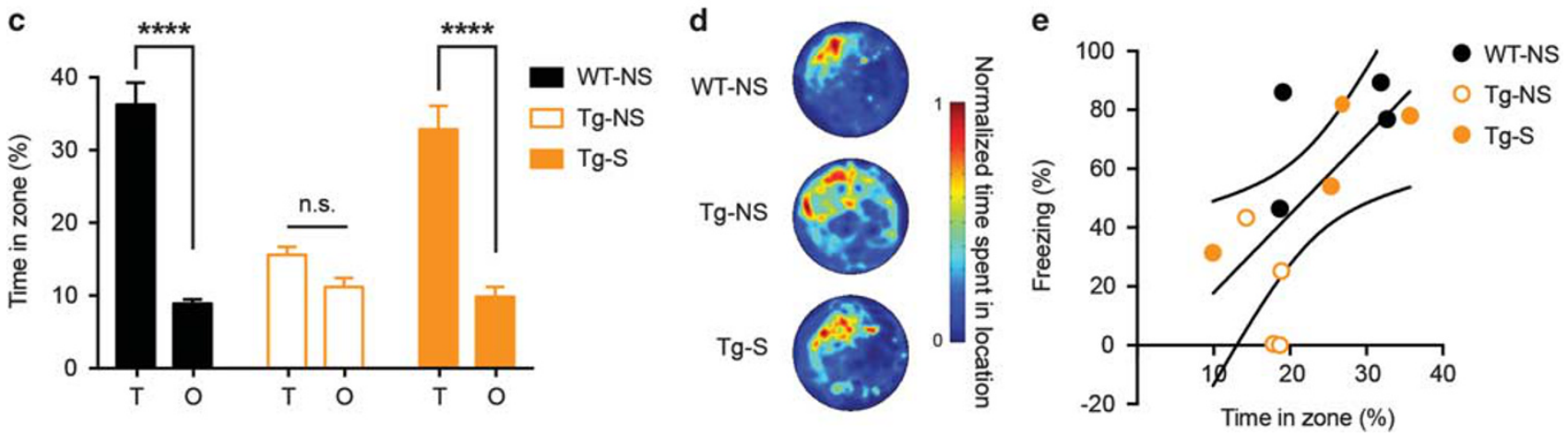

Figure 3 EC stimulation at 6 weeks of age rescues the spatial memory deficits in young Tg mice, similar to the context memory deficits. (a) Tg mice were bilaterally stimulated in the EC (Tg-S), whereas Tg and WT controls were not stimulated (Tg-NS, WT-NS) at 6 weeks of age. Mice were trained in the hidden platform version of the water maze and spatial memory probed at the completion of training. (b) All mice gradually showed decrease in latency to reach the platform over days, although WT mice were generally quicker, indicating that all groups learned the task (WT-NS, $n=18 ; \mathrm{Tg}-\mathrm{NS}, n=8$; Tg-S, $n=7$ ). (c, d) ECDBS rescued the spatial memory deficit in Tg mice. Tg-S and WT mice spent similar amounts of time in the zone that previously housed the platform (Target zone, T) than in other equal-sized zones in other areas of the pool (Other zones, O), whereas Tg-NS mice did not (WT-NS, $n=18$; Tg-NS, $n=8$; Tg-S, $n=7$ ). (e) Scatterplot showing performance for subsets of mice that were tested in both the contextual fear and water maze tasks. Overall, performance was positively correlated. However, within group, these correlations were not significant, most likely because the low sample sizes (WT-NS, $n=4$; Tg-NS, $n=4$; Tg-S, $n=4)$. Data are expressed as mean \pm SEM (*****P<0.000 I, n.s.: not significant).

previously observed rescue of contextual fear memory persisted. WT-NS and Tg-S again showed higher freezing levels than Tg-NS mice (Figure $2 c ; F_{2,29}=5.23, P<0.05$; WT-NS $(n=18)$, Tg-NS $(n=7), \operatorname{Tg}-\mathrm{S}(n=7) ;$ post hocs Newman-Keuls). This finding again confirms our original observations (Figure 1d), and indicates that the rescue of contextual fear memory in stimulated $\mathrm{Tg}$ mice is both specific (does not extend to tone fear memory) and persistent (for at least one week).

\section{EC Deep Brain Stimulation Reverses Spatial Learning Deficits in Young TgCRND8 Mice}

We next asked whether the beneficial effects of entorhinal cortex stimulation on contextual fear memory generalized to other forms of hippocampal-dependent memory. Similar to contextual fear conditioning, the hidden-platform version of the water maze also depends on the dorsal hippocampus (Morris et al, 1982). We focused on the 6 week post-EC stimulation time point, as this time point showed a strong rescue of contextual fear memory deficits in $\mathrm{Tg}$ mice (Figure 1d, Figure 3a).

Six weeks following EC stimulation, Tg-S, Tg-NS, and WT-NS mice were trained to locate a hidden platform in a fixed location over 6 training days ( 3 trials/day). Across training days, all mice located the platform more efficiently
(Figure 3b; significant main effect of Day $F_{5,150}=25.64$, $p<0.001$, but no Group $\times$ Day interaction in a mixed ANOVA; Tg-S $(n=7)$, Tg-NS $(n=8)$, WT-NS $(n=18))$. In general, WT-NS mice showed shorter escape latencies over training (significant main effect of Group, $F_{2,30}=5.23$, $P<0.05)$. Therefore, although WT mice showed generally better performance across training days, all mice showed evidence of learning.

Spatial memory was assessed in a probe test conducted $24 \mathrm{~h}$ after the completion of training. The platform was removed from the pool and the percent time mice spent in a circular target zone $(\mathrm{T}, 20 \mathrm{~cm}$ radius) centered on the platform location during training $v s$ time spent in similarly sized circular zones located in the other $(\mathrm{O}, 20 \mathrm{~cm}$ radius $)$ three quadrants of the pool was measured (Moser et al, 1993). Stimulated Tg mice showed spatial memory comparable to WT-NS mice, and considerably better than nonstimulated $\mathrm{Tg}$ mice (Figures $3 \mathrm{c}$ and d; significant Group $\times$ Zone interaction, $F_{2,30}=9.18, P<0.001$; post hoc analyses revealed WT-NS and Tg-S (but not Tg-NS) spent more time searching in the target $(\mathrm{T})$ zone compared to other $(\mathrm{O})$ zones, indicating robust spatial memory).

A cohort of mice was tested in both contextual fear conditioning and the water maze, allowing for within-subject comparison of performance across the two tasks. The correlation analysis was statistically underpowered when 
tested within each group owing to the small sample sizes ( $n=4$ for each group). However, there was an overall trend for performance on the two tasks to be positively correlated. This suggests that mice that exhibited stronger contextual fear memory likely performed better in the water maze probe test (Figure 3e; WT: $r=0.50, P=0.50$; Tg-S: $r=0.88, P=0.12$; Tg-NS: $r=0.73, P=0.27)$. Furthermore, when all the groups were combined, the performance in contextual fear conditioning and water maze tasks was correlated $(r=0.66$, $P<0.05)$.

Together these results suggest that the rescue by EC stimulation in $\mathrm{Tg}$ mice generalizes across hippocampusdependent types of memory but not to hippocampusindependent types of memory.

\section{EC Stimulation Reduces Plaque Load in Young Mice}

In TgCRND8 mice, A $\beta$ plaques emerge when mice are $\sim 10-$ 12 weeks of age and increase with time (Yiu et al, 2011). We next assessed whether EC stimulation impacts subsequent plaque load. After behavioral testing was complete in each group (mice trained at 1, 3, or 6 weeks following stimulation at 6 weeks of age), we examined the brains of Tg-S and Tg-NS mice for A $\beta$ plaques. As expected (Chishti et al, 2001, Yiu et al, 2011), negligible plaques were observed in either the hippocampus or cortex of WT mice or Tg mice trained 1 or 3 weeks following EC stimulation (at this time, Tg mice were 7 weeks and 9 weeks of age, respectively). At 12 weeks of age, Tg-NS $(n=7)$ mice showed substantial plaque load (Figure 4a). Strikingly, EC stimulation 6 weeks before (Tg-S, $n=11$ ) reduced plaque load in both the dorsal hippocampus (Figure $4 \mathrm{~b} ; t_{16}=2.36, P<0.05$ ) and cortex
(Figure $4 \mathrm{c} ; t_{16}=2.30, P<0.05$ ). This finding suggests one potential mechanism by which EC stimulation reverses the cognitive decline in the early stages of $\mathrm{AD}$.

\section{EC Stimulation Reverses Memory Deficits in Older TgCRND8 Mice}

As in $\mathrm{AD}$, the memory deficits and plaque load worsen in TgCRND8 mice with age (Hyde et al, 2005, Francis et al, 2012). Therefore, we next asked whether EC stimulation reverses the memory deficits in older $\mathrm{Tg}$ mice with more advanced stages of pathology.

Six-month-old Tg mice received bilateral EC stimulation for $1 \mathrm{~h}$, as above. Six weeks later, we trained mice ( $\mathrm{Tg}-\mathrm{S}$, Tg-NS, WT-NS) in contextual fear conditioning as above. Memory was assessed $24 \mathrm{~h}$ later (Figure 5a). Remarkably, similar to younger Tg mice, EC stimulation also rescued the contextual fear memory deficit in 6-month-old Tg mice (Figure 5b; $F_{2,45}=17.14, P<0.0001$; WT-NS $(n=25)$, Tg-NS $(n=10), \operatorname{Tg}-S(n=13))$. Similar to younger $\mathrm{Tg}$ mice, EC stimulation did not affect tone fear memory deficits in older Tg mice (Figures $5 \mathrm{c}$ and $\mathrm{d} ; F_{2,45}=30.22, P<0.0001$; WT-NS froze significantly more than $\mathrm{Tg}-\mathrm{NS}$ and $\mathrm{Tg}-\mathrm{S}$ mice which did not differ from each other, by Newman-Keuls post hocs). However, when these groups of mice were returned to the original training context one week later, once again Tg-S froze at high levels, similar to WT-NS mice, and higher than Tg-NS mice (Figure 5e; $F_{2,38}=8.63, P<0.001$; WT-NS $(n=20)$, Tg-NS $(n=10)$, Tg-S $(n=11)$; post hocs NewmanKeuls). This result in older $\mathrm{Tg}$ mice mirrors the EC stimulation rescue of memory deficits in younger $\mathrm{Tg}$ mice. That is, EC stimulation reverses contextual fear memory

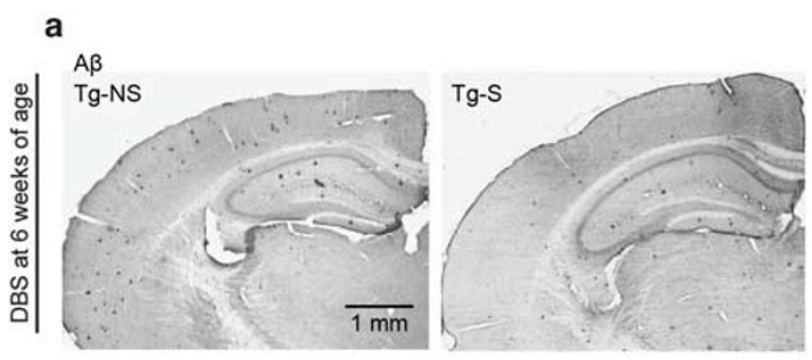

d

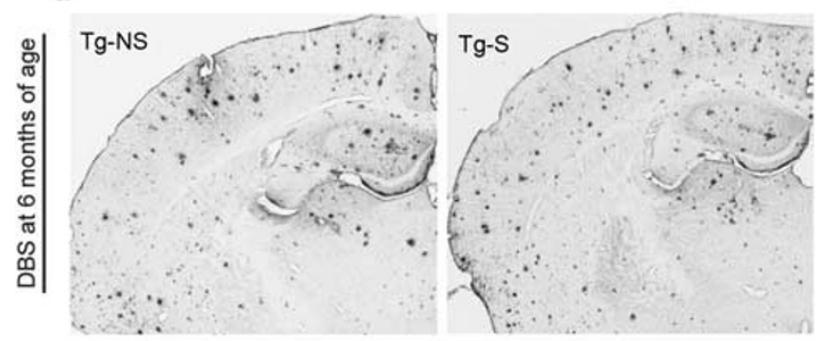

b

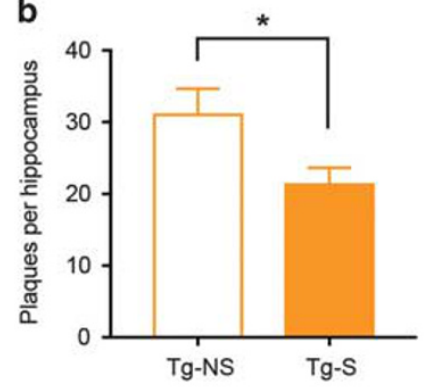

e

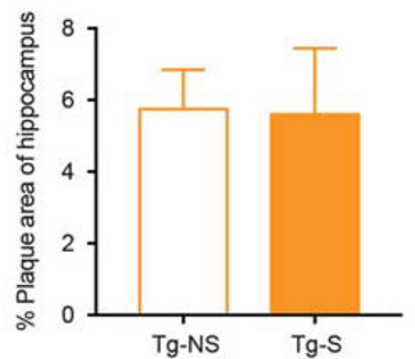

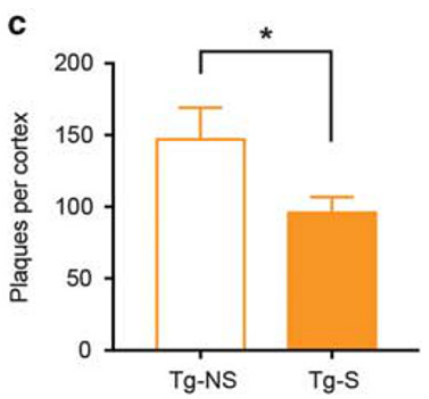

f

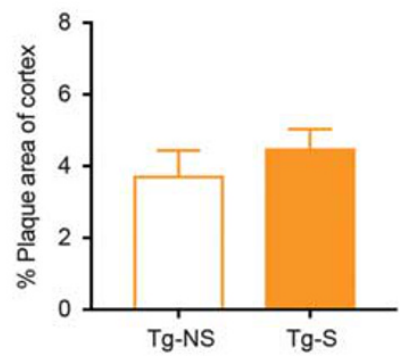

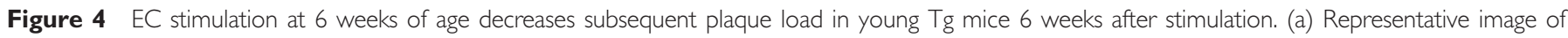

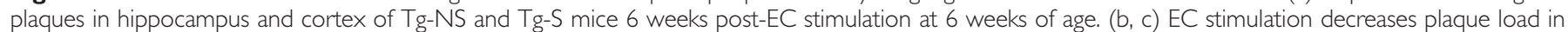

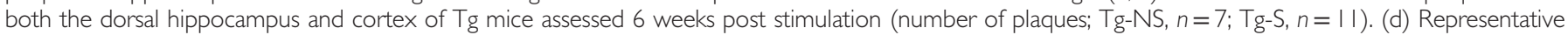

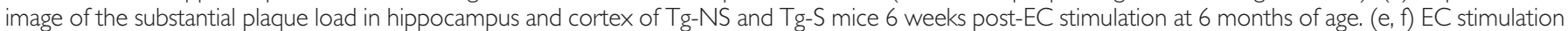

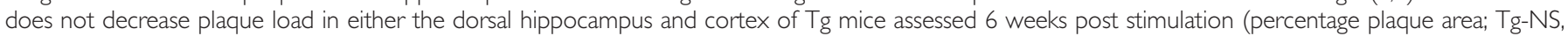
$n=7$; Tg-S, $n=r)$. Data are expressed as mean \pm SEM $(* P<0.05)$. 
deficits; this rescue is specific (does not generalize to tone fear memory) and persistent (at least 1 week).

To assess whether the beneficial effects of EC stimulation in older Tg mice generalize to other forms of hippocampusdependent learning, we next trained these mice in the water maze ( 6 days, 3 trials per day, as above, Figure 5f). Across training days, all groups of mice learned to locate the platform more efficiently (Figure 5g; Group $\times$ Day ANOVA; showing a significant effect of Day, $F_{5,220}=52.28, P<0.001$, suggesting mice improving over days, and a significant main effect of Group, $F_{2,44}=10.88, P<0.001$, reflecting generally shorter escape latencies in WT-NS $(n=22)$ group compared with either Tg-S $(n=14)$ or Tg-NS $(n=11)$ groups across days).

In the probe test conducted $24 \mathrm{~h}$ following the completion of training, stimulated $\mathrm{Tg}$ mice showed similar levels of spatial memory to WT-NS mice, and considerably better performance than non-stimulated $\mathrm{Tg}$ mice (Figures $5 \mathrm{~h}$ and i; Group $\times$ Zone interaction, $F_{2,44}=4.17, P<0.05$; post hoc analyses indicated WT-NS and Tg-S (but not Tg-NS) spent more time searching in the target $(\mathrm{T})$ zone compared with other (O) zones).

Similar to the 6 week group, a cohort of 6-month-old mice was tested in both contextual fear conditioning paradigm and the water maze. Strikingly, water maze performance and levels of contextual fear memory were significantly correlated in Tg-S mice (Figure 5j; $r=0.76, P<0.05 ; n=8$ ). This suggests that EC-DBS may be driving this correlated enhancement in performance across both tasks. This correlation was not observed in WT-NS (Supplementary Figure S1a; $r=0.04, P>0.05 ; n=14)$ or Tg-NS mice (Supplementary Figure S1b; $r=0.006, P>0.05 ; n=6$ ). The lack of correlation in these latter two groups is likely because mice were performing at ceiling levels (WT-NS) and floor levels (Tg-NS), respectively, potentially masking the relationship between performance in the two tasks.

As before, we also assessed whether EC stimulation impacted plaque load. After behavioral testing was complete, we examined the brains of Tg-S and Tg-NS mice for A $\beta$ plaques. In contrast to the younger mice, EC stimulation at six months of age (Figure $4 \mathrm{~d}$ ) did not produce a detectable decrease in plaque load in either the dorsal hippocampus (Figure $4 \mathrm{e} ; t_{9}=0.95, P>0.05$ ) or cortex (Figure 4f; $t_{9}=0.56$, $P>0.05)$. It is possible that the lack of impact on plaques could be due to substantial deposition already present at 6 months of age in the Tg mice.

\section{Contextual Fear and Water Maze Memory Rescue are Observed in Both Male and Female Tg Mice}

We next examined whether the ability of DBS to reverse memory deficits in Tg mice was sex-dependent. As DBS reversed memory deficits in 6-week-old and 6-month-old mice we combined these groups for our analysis, and analyzed contextual fear memory and water maze performance.

Interestingly, for contextual fear memory, we found freezing levels were higher in female compared to male mice. However, DBS treatment did not differentially impact freezing levels (Supplementary Figure S1c, d; males (WT-NS $(n=24)$, Tg-NS $(n=10)$, Tg-S $(n=15))$; females (WT-NS $(n=26), \quad$ Tg-NS $\quad(n=9), \quad$ Tg-S $\quad(n=14)) ; \quad$ Sex $\times$ Group
ANOVA; showing a significant main effect of Sex; $\mathrm{F}_{1,92}=7.66, \quad P<0.01$; but no significant main effect of Sex $\times$ Group interaction; $F_{2,92}=0.48, P>0.05$ ).

We performed a similar analysis on the water maze data. In this case, there was no difference in performance across males and females, nor did DBS differentially impact water maze performance across sexes (Supplementary Figure S1e-f; Males (WT-NS $(n=21)$, Tg-NS $(n=12)$, Tg-S $(n=15))$; Females (WT-NS $(n=19)$, Tg-NS $(n=7)$, Tg-S $(n=6))$; Sex $\times$ Group $\times$ Zone ANOVA; showing no significant main effect of Sex $\left(\mathrm{F}_{1,74}=0.1, P>0.05\right)$, and no significant two- or three-way Sex interactions (Sex $\times$ Group $\times$ Zone $\mathrm{F}_{2,74}=0.3, P>0.05$; Sex $\times$ Group $\mathrm{F}_{2,74}=0.6, P>0.05$; Sex $\times$ Zone $\left.\mathrm{F}_{1,74}=0.1, P>0.05\right)$ ).

\section{Contextual Fear Memory Rescue is Specific and Not Due to Increase in Anxiety-Like Behavior in Young and Old Tg Mice}

To verify that the observed rescue in contextual fear conditioning is specific and not due to increase in overall basal anxiety-like behavior perhaps caused by EC stimulation, we also assessed behavior in the open field (Supplementary Figure S2a) (Crawley et al, 1997, Prut and Belzung, 2003). In the open field, we observed no effect of EC stimulation on total distance traveled (Supplementary Figure S2b, no significant effect of Group $\times$ Distance, $F_{2,30}=1.11$, $P>0.05$; WT-NS $(n=18)$, Tg-NS $(n=8)$ and Tg-S $(n=7))$ or distance traveled in the periphery, middle, and center of the open field (Supplementary Figure S2c; no significant effect of Group $\times$ Zone (distance traveled in outer, middle, and inner zones of open field) $F_{4,60}=0.20, P>0.05$, or Group, $F_{2,30}=0.66, P>0.05$, but, as expected, significant effect of Zone, $F_{2,60}=96.93, P<0.001$, with all groups of mice traveling more distance in the outer zone).

Similarly in mice stimulated at 6 months of age, there was no difference found in total distance traveled (Supplementary Figure S2d; no significant effect of Group $\times$ Distance, $F_{2,20}=1.15, P>0.05 ;$ WT-NS $(n=11)$, Tg-NS $(n=6)$ and Tg-S $(n=6))$ and distance traveled in the periphery, middle, and center of the open field (Supplementary Figure S2e; no significant effect of Group $\times$ Zone (Distance traveled in outer, middle and inner zones of open field) $F_{4,40}=0.07, P>0.05$, or Group, $F_{2,30}=0.60$, $P>0.05$, but, as expected, significant effect of Zone, $F_{2,40}=23.43, P<0.001$, with all groups of mice traveling more distance in the outer zone). These results verify that the increase in freezing observed following EC stimulation cannot be attributed to a non-specific increase in anxietylike behavior.

\section{DISCUSSION}

Here we tested memory in TgCRND8 transgenic mice that express human APP with the Swedish and Indiana FAD mutations. These mice express high levels of $A \beta$, show progressive, age-related deficits in spatial and contextual fear memory and amyloid plaque deposition (Chishti et al, 2001, Yiu et al, 2011). We find that a single DBS treatment of the EC at 6 weeks of age is sufficient to rescue the contextual fear and spatial memory deficits in $\mathrm{Tg}$ mice trained 3 weeks or 
6 weeks after stimulation. This memory rescue was specific to hippocampal-dependent forms of memory and did not extend to auditory fear conditioning. In addition, DBS was equally effective in both male and female mice, and anxiety-like or general locomotor behavior was unaffected by stimulation. Importantly, DBS also decreased subsequent plaque load in the hippocampus and cortex in mice trained 6 weeks after stimulation. Therefore, in young Tg mice, acute

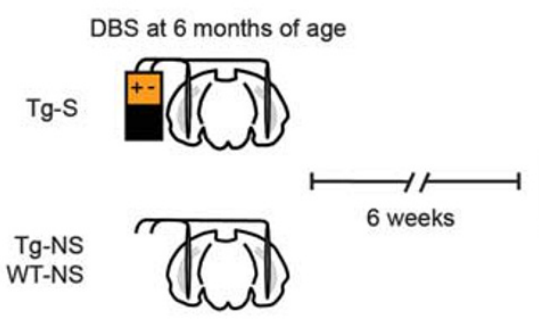

C

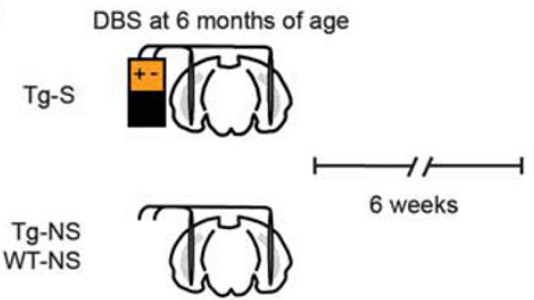

d

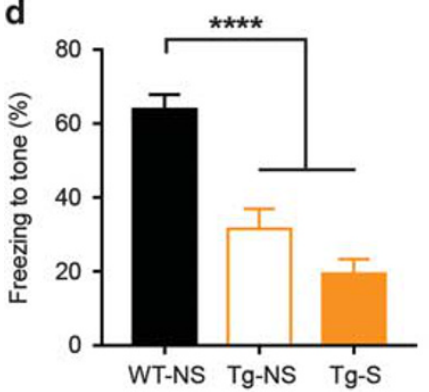

f

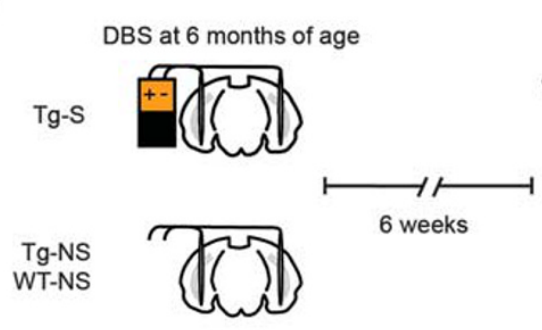

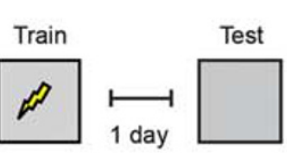

1 day
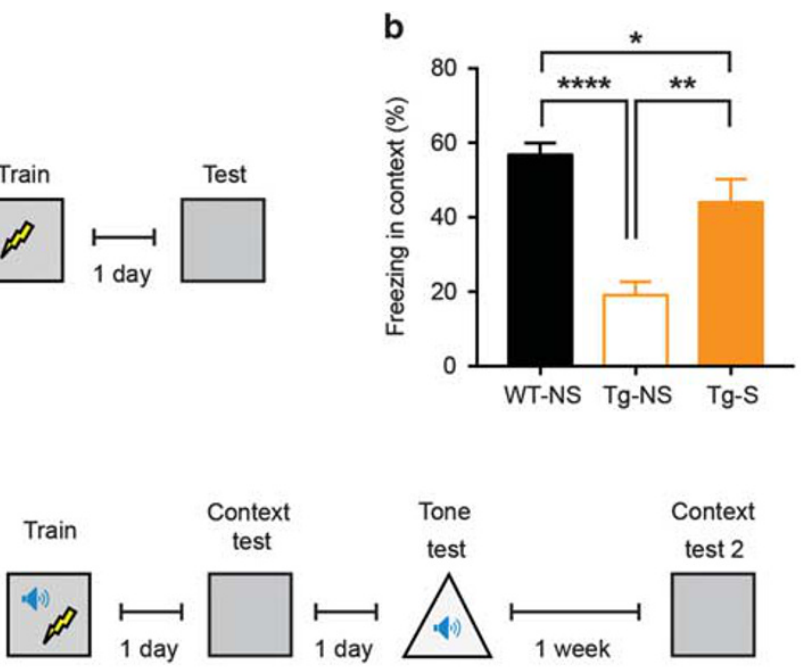

e

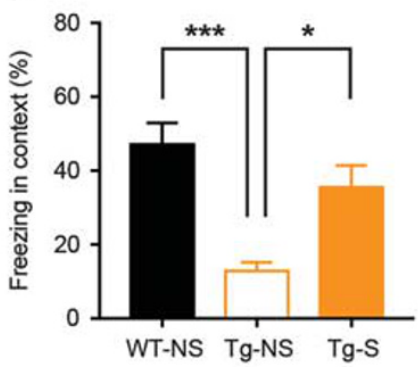

g

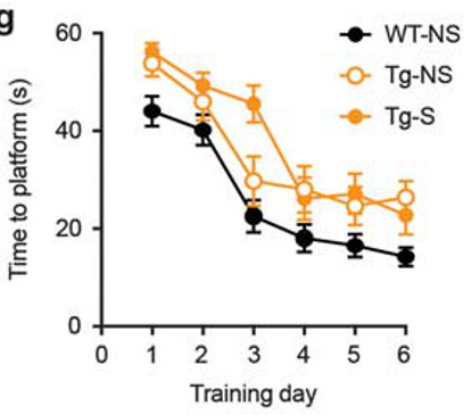

h

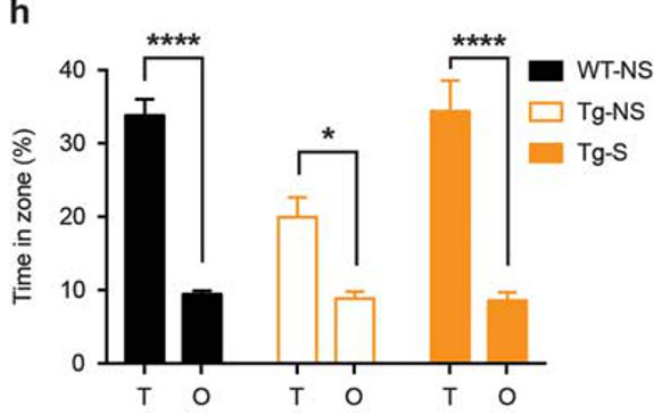

i

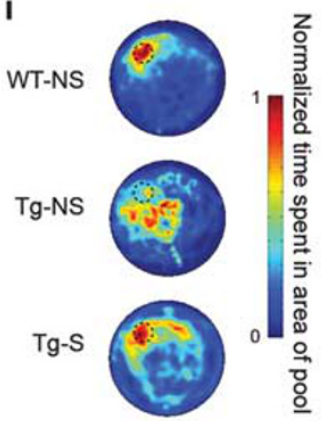

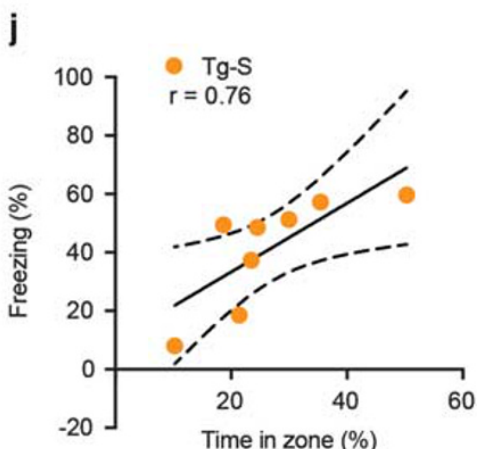


stimulation was sufficient to overcome hippocampaldependent memory effects.

In young (6 week-old) Tg mice, the DBS-mediated reversal in memory deficits only became apparent 3-6 weeks post stimulation and was not detected 1 week post stimulation. One possibility for the lack of rescue at 1 week post stimulation is that mice at this age ( 7 weeks old) generally froze less than those tested at 6 weeks post stimulation (12 weeks old). The increase in memory performance with age is consistent with previous studies from our group (Akers et al, 2012) and others (Smith et al, 2006). However, we do not think that age-dependent changes in freezing behavior confound the interpretation of the DBS effects. The control (WT-NS, Tg-NS) mice tested 1 or 3 weeks post stimulation exhibited similar levels of freezing that were both lower than the mice tested 6 weeks post-DBS. Nevertheless, DBS still rescued contextual fear memory in the mice tested 3 weeks poststimulation, similar to the rescue observed in the 6 week post-DBS group. This indicates that DBS was effective regardless of whether baseline freezing levels were low ( 3 weeks post DBS) or high (6 weeks post DBS), and the rescue was a gradual process requiring at least 3-6 weeks.

As Tg mice show progressive pathology over time, we also tested whether the memory deficits in older (6-month-old) Tg mice could be reversed by acute DBS. Strikingly, DBS treatment at 6 months of age also restored contextual fear and spatial memory deficits assessed 6 weeks after treatment. Again, memory restoration was equally effective in both male and female mice, and specific to memories critically dependent on the hippocampus, as an amygdala-dependent memory was not restored. Interestingly, the performance of Tg-S, but not WT-NS or Tg-NS, mice in water maze and contextual fear conditioning were highly correlated, suggesting that DBS drove the enhancement in performance across both tasks. However, it is difficult to discern whether the performance in these two tasks is correlated in the other groups (WT-NS, Tg-NS), because of the near-ceiling, or floor, memory performance in these mice, respectively. As with the 6 week group, other behavioral measures such as anxiety were not impacted, showing the specificity of the observed effect.

What is (are) the mechanism(s) of the pro-cognitive effects of EC-DBS? Although we did not address potential mechanisms in the present study, we did note that the memory improvement produced by EC stimulation gradually appeared in the weeks following stimulation. That is, the memory rescue was not apparent 1 week following stimulation, but emerged 3-6 weeks post stimulation. One possible mechanism is via reduction in plaque load. We observed decreased plaque load following DBS in young (6-week-old) mice, in both the hippocampus and cortex. The reduction of hippocampal plaques may be mediated by direct projections from the entorhinal cortex to hippocampus (eg, CA1 and dentate gyrus (Witter et al, 1988)). The reduction in cortical plaques may be mediated by direct projections to the prefrontal cortex, amygdala (Kitamura et al, 2017), and indirect projections to the cortex via the hippocampus, and/or striatum (Totterdell and Meredith, 1997). In addition, we note that DBS of the fornix in patients also impacted cortex (ie, increased cortical glucose metabolism and cortical volume; (Sankar et al, 2015, Rowland et al, 2016)), suggesting that DBS likely mediates its effects by rescuing dysfunctions of brain-wide circuits. Therefore, although we did not observe reversal of amygdala-dependent memory (ie, auditory fear memory), it is possible that other corticaldependent mnemonic abilities (eg, remote memory, conditioned taste aversion) may be rescued by DBS. Further studies are needed to explore these possibilities.

Interestingly, in contrast to the young mice, DBS did not reduce plaque load in 6-month-old mice, despite successful rescue in memory deficits. This might suggest that DBS may act through a plaque-dependent mechanism only in early stage of $\mathrm{AD}$. Alternatively, plaque load reduction may not be the primary mechanism by which DBS reverses memory deficits. This is consistent with a number of observations including: 1) memory deficits emerge before plaques in this AD mouse model (at 1 and 3 weeks post stimulation, Tg-NS mice (7-9 weeks old) show memory deficits in contextual fear conditioning, but no detectable levels of plaque); (2) similar dissociations between memory loss and plaque load have been reported in patients; (3) immunization against $A \beta$ rescues memory deficits in Alzheimer's mouse model without affecting plaque load (Dodart et al, 2002).

An additional mechanism of EC-DBS may be the promotion of hippocampal neurogenesis. We previously showed that in WT mice, the same EC-DBS promotes activity-dependent neurogenesis of hippocampal neurons to enhance spatial memory (Stone et al, 2011). Specifically, we previously showed that the same EC-DBS enhanced the production and survival of newborn neurons in the dentate gyrus, a region that receives direct EC input. Moreover, these EC-DBS-induced newborn neurons become functionally equivalent to developmentally generated neurons in the DG. Finally, blocking neurogenesis also blocked the memory 
enhancing effects of EC-DBS. Similarly, an alternative approach of enhancing neurogenesis via lithium was also found to rescue memory deficits in a transgenic $\mathrm{AD}$ mouse model (Fiorentini et al, 2010).

Although these lines of evidence suggest that the procognitive effects of EC-DBS are at least partly mediated by reduction in plaques and enhancement in adult hippocampal neurogenesis, they do not preclude the involvement of other mechanisms. For instance, DBS has been proposed to excite or inhibit local neurons (Kringelbach et al, 2007, Chiken and Nambu, 2016), modulate neurotransmission (Chiken and Nambu, 2016), restore disrupted circuit activity (Mayberg et al, 2005, Laxton et al, 2010), or synchronize brain activity (Murrow, 2014). Further studies are required to address these questions.

There are currently only two approved pharmacological treatments for $\mathrm{AD}$. Recent clinical trials with pharmacological agents and vaccine targeting of $\mathrm{A}$ have been disappointing (Winblad et al, 2016). The current results showing the beneficial effects of an 'electroceutical' intervention in a mouse model of $\mathrm{AD}$ further strengthen the pursuit of these types of interventions.

\section{FUNDING AND DISCLOSURE}

This work was supported by Canadian Institutes of Health Research (CIHR) grants to PWF (FDN143227) and SAJ (MOP74650). PWF and SAJ hold Canada Research Chairs, and are senior fellows in the Canadian Institute for Advanced Research (CIFAR) programs in Child and Brain Development and Brain, Mind and Consciousness programs, respectively. FX was supported by fellowships from the Natural Sciences and Engineering Research Council of Canada (NSERC) and CIHR. The authors declare no conflict of interest.

\section{REFERENCES}

Akers KG, Arruda-Carvalho M, Josselyn SA, Frankland PW (2012). Ontogeny of contextual fear memory formation, specificity, and persistence in mice. Learn Mem 19: 598-604.

Albert MS (2011). Changes in cognition. Neurobiol Aging 32(Suppl 1): S58-S63.

Blanchard RJ, Blanchard DC (1969). Crouching as an index of fear. J Compar Physiol Psychol 67: 370-375.

Chiken S, Nambu A (2016). Mechanism of deep brain stimulation: inhibition, excitation, or disruption? Neuroscientist 22: 313-322.

Chishti MA, Yang DS, Janus C, Phinney AL, Horne P, Pearson J et al (2001). Early-onset amyloid deposition and cognitive deficits in transgenic mice expressing a double mutant form of amyloid precursor protein 695. J Biol Chem 276: 21562-21570.

Citron M, Oltersdorf T, Haass C, McConlogue L, Hung AY, Seubert P et al (1992). Mutation of the beta-amyloid precursor protein in familial Alzheimer's disease increases beta-protein production. Nature 360: 672-674.

Crawley JN, Belknap JK, Collins A, Crabbe JC, Frankel W, Henderson $\mathrm{N}$ et al (1997). Behavioral phenotypes of inbred mouse strains: implications and recommendations for molecular studies. Psychopharmacology 132: 107-124.

Dodart JC, Bales KR, Gannon KS, Greene SJ, DeMattos RB, Mathis C et al (2002). Immunization reverses memory deficits without reducing brain Abeta burden in Alzheimer's disease model. Nat Neurosci 5: 452-457.
Fiorentini A, Rosi MC, Grossi C, Luccarini I, Casamenti F (2010). Lithium improves hippocampal neurogenesis, neuropathology and cognitive functions in APP mutant mice. PloS ONE 5: e14382.

Francis BM, Kim J, Barakat ME, Fraenkl S, Yucel YH, Peng S et al (2012). Object recognition memory and BDNF expression are reduced in young TgCRND8 mice. Neurobiol Aging 33: 555-563.

Hamani C, McAndrews MP, Cohn M, Oh M, Zumsteg D, Shapiro $\mathrm{CM}$ et al (2008). Memory enhancement induced by hypothalamic/fornix deep brain stimulation. Ann Neurol 63: 119-123.

Hardy J, Selkoe DJ (2002). The amyloid hypothesis of Alzheimer's disease: progress and problems on the road to therapeutics. Science 297: 353-356.

Hyde LA, Kazdoba TM, Grilli M, Lozza G, Brusa R, Zhang Q et al (2005). Age-progressing cognitive impairments and neuropathology in transgenic CRND8 mice. Behav Brain Res 160: 344-355.

Kim JJ, Fanselow MS (1992). Modality-specific retrograde amnesia of fear. Science 256: 675-677.

Kitamura T, Ogawa SK, Roy DS, Okuyama T, Morrissey MD, Smith LM et al (2017). Engrams and circuits crucial for ssytems consoldiation of a memory. Science 356: $73-78$ in press.

Kringelbach ML, Jenkinson N, Owen SL, Aziz TZ (2007). Translational principles of deep brain stimulation. Nat Rev Neurosci 8: 623-635.

Laxton AW, Tang-Wai DF, McAndrews MP, Zumsteg D, Wennberg R, Keren R et al (2010). A phase I trial of deep brain stimulation of memory circuits in Alzheimer's disease. Ann Neurol 68: 521-534.

Lozano AM, Fosdick L, Chakravarty MM, Leoutsakos JM, Munro C, Oh E et al (2016). A Phase II Study of Fornix Deep Brain Stimulation in Mild Alzheimer's Disease. J Alzheimers Dis 54: 777-787.

Maei HR, Zaslavsky K, Teixeira CM, Frankland PW (2009). What is the most sensitive measure of water maze probe test performance? Front Integr Neurosci 3: 4.

Mayberg HS, Lozano AM, Voon V, McNeely HE, Seminowicz D, Hamani C et al (2005). Deep brain stimulation for treatmentresistant depression. Neuron 45: 651-660.

Morris GP, Clark IA, Vissel B (2014). Inconsistencies and controversies surrounding the amyloid hypothesis of Alzheimer's disease. Acta Neuropathol Commun 2: 135.

Morris RG, Garrud P, Rawlins JN, O'Keefe J (1982). Place navigation impaired in rats with hippocampal lesions. Nature 297: 681-683.

Moser E, Moser MB, Andersen P (1993). Spatial learning impairment parallels the magnitude of dorsal hippocampal lesions, but is hardly present following ventral lesions. J Neuroscience 13: 3916-3925.

Murrow RW (2014). Penfield's prediction: a mechanism for deep brain stimulation. Front Neurol 5: 213.

Paxinos G, Franklin KBJ (2001). The Mouse Brain in Stereotaxic Coordinates2nd Edn.Academic Press: San Diego.

Perry RJ, Hodges JR (1996). Spectrum of memory dysfunction in degenerative disease. Curr Opin Neurol 9: 281-285.

Ponce FA, Lozano AM (2010). Deep brain stimulation state of the art and novel stimulation targets. Prog Brain Res 184: 311-324.

Price DL, Sisodia SS (1998). Mutant genes in familial Alzheimer's disease and transgenic models. Annu Rev Neurosci 21: 479-505.

Prince M, Comas-Herrera A, Knapp M, Guerchet M, Karagiannidou M (2016). World Alzheimer Report 2016. Alzheimer's Disease International (ADI): London.

Prut L, Belzung C (2003). The open field as a paradigm to measure the effects of drugs on anxiety-like behaviors: a review. Eur $J$ Pharmacol 463: 3-33.

Rowland NC, Sammartino F, Tomaszczyk JC, Lozano AM (2016). Deep brain stimulation of the fornix: engaging therapeutic circuits and networks in Alzheimer disease. Neurosurgery 63 (Suppl 1): 1-5. 
Sankar T, Chakravarty MM, Bescos A, Lara M, Obuchi T, Laxton AW et al (2015). Deep brain stimulation influences brain structure in Alzheimer's disease. Brain Stimul 8: 645-654.

Selkoe DJ (2002). Alzheimer's disease is a synaptic failure. Science 298: 789-791.

Smith LN, McDonald CG, Bergstrom HC, Brielmaier JM, Eppolito AK, Wheeler TL et al (2006). Long-term changes in fear conditioning and anxiety-like behavior following nicotine exposure in adult versus adolescent rats. Pharmacol, Biochem, Behav 85: 91-97.

Steele JW, Brautigam H, Short JA, Sowa A, Shi M, Yadav A et al (2014). Early fear memory defects are associated with altered synaptic plasticity and molecular architecture in the TgCRND8 Alzheimer's disease mouse model. J Compar Neurol 522: 2319-2335.

Stone SS, Teixeira CM, Devito LM, Zaslavsky K, Josselyn SA, Lozano AM et al (2011). Stimulation of entorhinal cortex promotes adult neurogenesis and facilitates spatial memory. J Neuroscience 31: 13469-13484.

Tanzi RE, Bertram L (2001). New frontiers in Alzheimer's disease genetics. Neuron 32: 181-184.
Teixeira CM, Pomedli SR, Maei HR, Kee N, Frankland PW (2006). Involvement of the anterior cingulate cortex in the expression of remote spatial memory. J Neurosci 26: 7555-7564.

Totterdell S, Meredith GE (1997). Topographical organization of projections from the entorhinal cortex to the striatum of the rat. Neuroscience 78: 715-729.

Volkmann J, Moro E, Pahwa R (2006). Basic algorithms for the programming of deep brain stimulation in Parkinson's disease. Mov Disord 21(Suppl 14): S284-S289.

Winblad B, Amouyel P, Andrieu S, Ballard C, Brayne C, Brodaty H et al (2016). Defeating Alzheimer's disease and other dementias: a priority for European science and society. Lancet Neurol 15: 455-532.

Witter MP, Griffioen AW, Jorritsma-Byham B, Krijnen JL (1988). Entorhinal projections to the hippocampal CA1 region in the rat: an underestimated pathway. Neurosci Lett 85 193-198.

Yiu AP, Rashid AJ, Josselyn SA (2011). Increasing CREB function in the CA1 region of dorsal hippocampus rescues the spatial memory deficits in a mouse model of Alzheimer's disease. Neuropsychopharmacology 36: 2169-2186.

Supplementary Information accompanies the paper on the Neuropsychopharmacology website (http://www.nature.com/npp) 